\title{
Aminopeptidases trim Xaa-Pro proteins, initiating their degradation by the Pro/N-degron pathway
}

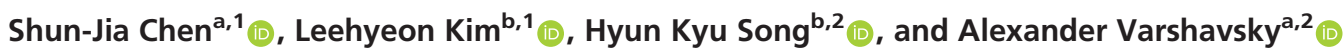

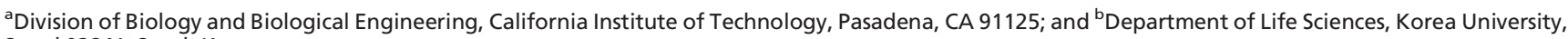 \\ Seoul 02841, South Korea \\ Contributed by Alexander Varshavsky, September 8, 2021 (sent for review August 21, 2021; reviewed by Ulrich Hartl and William P. Tansey)
}

\begin{abstract}
$\mathrm{N}$-degron pathways are proteolytic systems that recognize proteins bearing $\mathrm{N}$-terminal ( $\mathrm{Nt}$ ) degradation signals (degrons) called $\mathrm{N}$-degrons. Our previous work identified Gid4 as a recognition component (N-recognin) of the Saccharomyces cerevisiae proteolytic system termed the proline (Pro)/N-degron pathway. Gid4 is a subunit of the oligomeric glucose-induced degradation (GID) ubiquitin ligase. Gid4 targets proteins through the binding to their Nt-Pro residue. Gid4 is also required for degradation of Nt-Xaa-Pro (Xaa is any amino acid residue) proteins such as Nt-[Ala-Pro]-Aro10 and $\mathrm{Nt}$-[Ser-Pro]-Pck1, with Pro at position 2. Here, we show that specific aminopeptidases function as components of the Pro/Ndegron pathway by removing $\mathrm{Nt}-\mathrm{Ala}$ or $\mathrm{Nt}$-Ser and yielding Nt-Pro, which can be recognized by Gid4-GID. Nt-Ala is removed by the previously uncharacterized aminopeptidase Fra1. The enzymatic activity of Fra1 is shown to be essential for the GIDdependent degradation of Nt-[Ala-Pro]-Aro10. Fra1 can also trim Nt-[Ala-Pro-Pro-Pro] (stopping immediately before the last Pro) and thereby can target for degradation a protein bearing this $\mathrm{Nt}$ sequence. Nt-Ser is removed largely by the mitochondrial/cytosolic/nuclear aminopeptidase Icp55. These advances are relevant to eukaryotes from fungi to animals and plants, as Fra1, Icp55, and the GID ubiquitin ligase are conserved in evolution. In addition to discovering the mechanism of targeting of Xaa-Pro proteins, these insights have also expanded the diversity of substrates of the Pro/ $\mathrm{N}$-degron pathway.
\end{abstract}

degron | ubiquitin | aminopeptidase | Fra1 | Icp55

ntracellular protein degradation protects cells from misfolded and other abnormal proteins, generates free amino acids for protein synthesis, and also regulates the levels of proteins that evolved to be constitutively or conditionally shortlived in vivo. Two major sets of intracellular proteolytic pathways are the ubiquitin (Ub)proteasome system and the autophagy-endosome-lysosome system. Chaperone proteins are an essential part of both systems (1-8). Ub ligases recognize their substrate proteins through specific degradation signals (degrons) and conjugate $\mathrm{Ub}$, a 9-kDa protein (usually in the form of a poly-Ub chain), to cognate amino acid residues (usually an internal lysine or lysines) of targeted substrates. Deubiquitylases mediate the removal of $\mathrm{Ub}$ from Ub-conjugated proteins (1-4, 9-13). Polyubiquitylated proteins can be recognized by the $26 \mathrm{~S}$ proteasome, an adenosine triphosphate-dependent multisubunit protease. This protease unfolds the targeted protein and processively destroys it to peptides that vary in size from $\sim 3$ to $\sim 25$ residues (14-19).

$\mathrm{N}$-degron pathways (they were previously called "N-end rule pathways") are a distinct class of processive proteolytic systems that have in common their ability to recognize proteins that contain N-terminal (Nt) degrons called N-degrons (20). Degradation of proteins targeted by $\mathrm{N}$-degron pathways is mediated by the $26 \mathrm{~S}$ proteasome and/or autophagy in eukaryotes and by the proteasome-like ClpAP protease in bacteria (SI Appendix, Fig. S1) (2, 20-60). Determinants of a eukaryotic N-degron include a destabilizing Nt-residue of a protein and its internal Lys residue(s) that acts as a site of polyubiquitylation $(20,22$, $53,56)$.
In eukaryotes, the Arg/N-degron pathway targets, in particular, specific unacetylated Nt-residues. The Ac/N-degron pathway targets, in particular, the $\mathrm{N}^{\alpha}$-terminally acetylated $\mathrm{Nt}$-residues. The proline (Pro)/N-degron pathway targets, in particular, the Nt-Pro residue. The Gly/N-degron pathway targets unmodified Nt-Gly, and the fMet/N-degron pathway targets Nt-formylated proteins (SI Appendix, Fig. S1) (20-56).

Most N-degrons are initially not active (preN-degrons). Active N-degrons are produced either constitutively (e.g., cotranslationally) or via regulated steps. A variety of nonprocessive proteases, including caspases, calpains, separases, and cathepsins, functions as "upstream" components of N-degron pathways since a cleavage of a protein can produce a C-terminal $(\mathrm{Ct})$ fragment bearing a destabilizing Nt-residue (i.e., an Nt-residue that can be recognized by a specific $\mathrm{N}$-degron pathway). Active $\mathrm{N}$-degrons can also be formed through Nt-acetylation, Nt-deamidation, Nt-oxidation, Ntarginylation, Nt-leucylation, and Nt-formylation (SI Appendix, Fig. S1). Recognition components of N-degron pathways, called $\mathrm{N}$-recognins, are E3 Ub ligases or other proteins (e.g., p62 in mammals or ClpS in bacteria) that can recognize N-degrons. All 20 amino acids of the genetic code have been shown to function, in cognate sequence contexts, as destabilizing Ntresidues (SI Appendix, Fig. S1) (refs. 20-56 and references therein).

Regulated degradation of proteins and their natural fragments by $\mathrm{N}$-degron pathways has been shown to mediate an

\section{Significance}

The Gid4 protein of the yeast proline (Pro)/N-degron pathway targets proteins for degradation by recognizing their $\mathrm{N}$-terminal (Nt) Pro residue. This proteolytic pathway can also target proteins that bear Nt-Pro at position 2 instead of position 1 . In the present study, we used genetic and biochemical approaches to discover that Fra1 and Icp55 are specific aminopeptidases that trim the Nt-Ala (A) and Nt-Ser (S) residues from AP-Aro10 and SP-Pck1 proteins, respectively, thereby yielding Nt-Pro and initiating degradation of these proteins by the Pro/N-degron pathway. In addition to discovering the mechanism of targeting of XP proteins, these insights have also expanded the diversity of substrates of the Pro/N-degron pathway.

Author contributions: S.-J.C., L.K., H.K.S., and A.V. designed research; S.-J.C. and L.K. performed research; S.-J.C., L.K., H.K.S., and A.V. analyzed data; and S.-J.C., L.K., H.K.S., and A.V. wrote the paper.

Reviewers: U.H., Max Planck Institute of Biochemistry; and W.P.T., Vanderbilt University.

The authors declare no competing interest.

Published under the PNAS license.

${ }^{1}$ S.J.C. and L.K. contributed equally to this work.

${ }^{2}$ To whom correspondence may be addressed. Email: hksong@korea.ac.kr or avarsh@ caltech.edu.

This article contains supporting information online at http://www.pnas.org/lookup/ suppl/doi:10.1073/pnas.2115430118/-/DCSupplemental.

Published October 18, 2021 
immense range of biological processes, including the sensing of oxygen, nitric oxide, heme, and short peptides; the control of peptide transport and subunit stoichiometries in protein complexes; selective elimination of misfolded or retro-translocated proteins; a suppression of neurodegeneration and regulation of apoptosis; the control of transcription, replication, DNA repair, and chromosome cohesion/segregation; the regulation of $G$ proteins, chaperones, cytoskeletal proteins, gluconeogenesis, autophagy, peptide transport, meiosis, circadian rhythms, cell migration, fat metabolism, cardiovascular system, the adaptive and innate immunity, neurogenesis, and spermatogenesis; and many processes in plants (refs. 20-60 and references therein).

Our previous work has shown that the Saccharomyces cerevisiae Gid4 functions as a Pro/N-recognin of the Pro/N-degron pathway (SI Appendix, Fig. S1E). Gid4, a subunit of the Ub ligase called GID (glucose-induced degradation), binds to the Nt-Pro (P) residue of a substrate protein (38). Targeting by Gid4 leads to the GID/proteasome-mediated degradation of, in particular, gluconeogenic enzymes P-Fbp1, P-Mdh2, and P-Icl1, which bear Nt-Pro (after the cotranslational removal of the initial Nt-Met residue) $(38-46,61)$. The yeast Pro/N-degron pathway also targets $\mathrm{Nt}$-[Xaa-Pro] proteins (Xaa denotes any residue), including 2-oxo-acid decarboxylase AP-Aro10 (Nt-[Ala-Pro]-Aro10) and the gluconeogenic enzyme phosphoenolpyruvate carboxykinase SP-Pck1 (Nt-[Ser-Pro]-Pck1), although these proteins bear Pro at position $2(38,46)$. (Positions are numbered after removed Nt-Met.)

Described here is the discovery that the aminopeptidases Fra1 and Icp55 trim Nt-[Xaa-Pro] proteins, yielding the Nt-Pro residue and initiating degradation of these proteins by the Pro/ N-degron pathway (Figs. 1-6 and SI Appendix, Figs. S1-S4). These advances are relevant to all eukaryotes, as Fra1, Icp55, and the GID Ub ligase are conserved in evolution. In addition to discovering the mechanism of targeting of Xaa-Pro proteins, these insights have also expanded the diversity of substrates of the Pro/N-degron pathway.

\section{Results and Discussion}

Nt-Sequencing of AP-Aro10 and SP-Pck1 Proteins. In our initial approach, Ct-triple-flagged $\mathbf{A P}-A r o 10_{3 f}$ and $\mathbf{S P}-\mathbf{P c k} 1_{3 \mathrm{f}}$ were expressed in $S$. cerevisiae, then isolated, and $\mathrm{Nt}$-sequenced using the Edman technique (62), yielding two nested Ntsequences for each of two proteins. One sequence was the unprocessed Nt-segment (after the removal of Nt-Met), while the other was a further trimmed segment bearing Nt-Pro. The relative levels of (trimmed) $\mathbf{P}$-Pck $1_{3 \mathrm{f}}$ and $\mathbf{P}$-Aro $10_{3 \mathrm{f}}$ were $\sim 50$ and $\sim 53 \%$, respectively (SI Appendix, Fig. S2 $A$ and $B$ ). The observed approximate percentages of (trimmed) P-Pck $1_{3 \mathrm{f}}$ and $\mathbf{P}$-Aro $10_{3 \mathrm{f}}$ are underestimates, as these P-proteins (in contrast to their XP-precursors) would be underrepresented at steady state, owing to their degradation by the Pro/N-degron pathway (Fig. 6 and SI Appendix, Fig. S2 $A$ and $B$ ). Although semiquantitative [owing to limitations of Nt-sequencing using the Edman method (62)], these findings (SI Appendix, Fig. S2 $A$ and $B$ ) were essential, as they initiated genetic and biochemical approaches of the present study by demonstrating the in vivo trimming of AP-Aro $10_{3 \mathrm{f}}$ and SP-Pck $1_{3 \mathrm{f}}$.

Fra1 Aminopeptidase Is Essential for the In Vivo Degradation of AP-Aro10. Our degradation assays used the promoter reference technique (PRT), in which a long-lived protein (dihydrofolate reductase $[\mathrm{DHFR}])$ and a test protein are expressed from two identical $\mathrm{P}_{T D H 3}$ promoters containing additional DNA elements (63). In a messenger RNA (mRNA), these elements form $5^{\prime}$ aptamers that can bind to tetracycline (Tc) upon its uptake by a cell. The binding of Tc halts translation of just two mRNAs and thereby, allows chase-degradation assays that bypass the use of
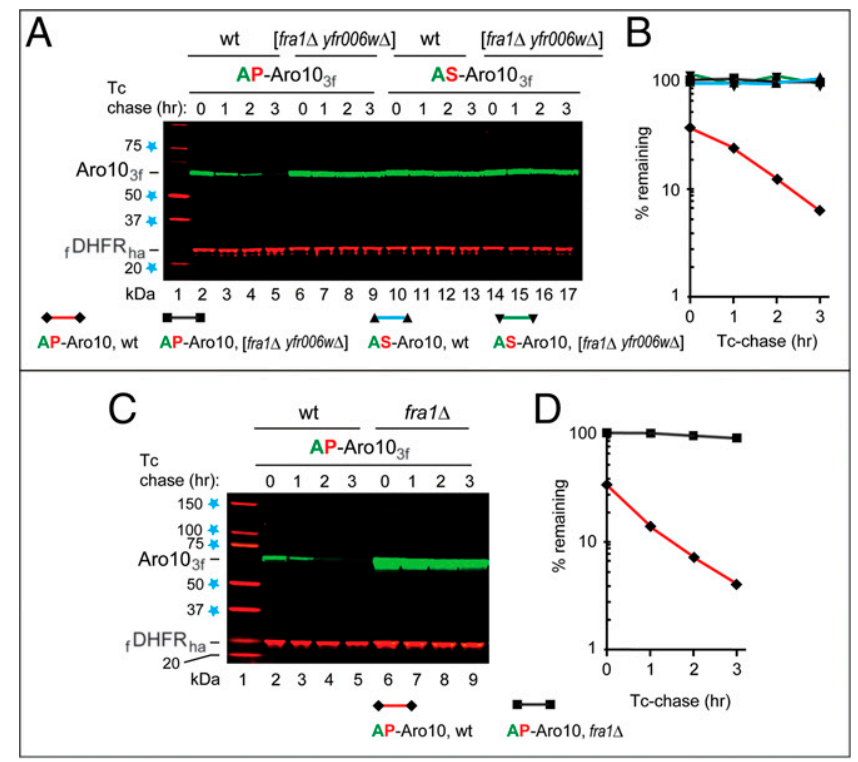

Fig. 1. Degradation of AP-Aro10 requires Fra1 aminopeptidase and Pro at position 2. (A) Lane 1, kilodalton markers. Lanes 2 to 5, Tc/PRT chase (SI Appendix, Fig. S2C) of AP-Aro $10_{3 f}$ at $30^{\circ} \mathrm{C}$ in wild-type (wt) cells. Lanes 6 to 9 , the same as lanes 2 to 5 but in double-mutant [fra $1 \Delta$ yfro06w $\Delta$ ] cells. Lanes 10 to 13 , the same as lanes 2 to 5 but with AS-Aro10 ${ }_{3 f}$. Lanes 14 to 17 , the same as lanes 6 to 9 but with AS-Aro10 ${ }_{3 \cdot}$. $(B)$ Quantification of data in A. (C) Lane 1, kilodalton markers. Lanes 2 to 5, TC/PRT chase of APAro $10_{3 f}$ in wt cells. Lanes 6 to 9 , the same as lanes 2 to 5 but in fra $1 \Delta$ cells. (D) Quantification of data in C. Regarding the absence of SD bars in $B$ and $D$ : shown are the degradation curves for the actual data in $A$ and $C$. All TCl PRT chases of this study were performed at least twice (usually thrice), with results differing by $10 \%$ or less. Blue stars on the left denote kilodalton markers.

global translation inhibitors. DHFR reference increases the accuracy of Tc/PRT since relative levels of a test protein can be measured as its ratios to the reference (63) (SI Appendix, Fig. S2C).

Degradation of $\mathbf{S P}-\mathbf{P c k} 1_{3 \mathrm{f}}$ was found to be slower than that of P-Pck $1_{3 f}$, the engineered Nt-Pro-bearing derivative of SP$\mathrm{Pck}_{3 \mathrm{f}}$ (SI Appendix, Fig. S2 D, lanes 2 to 5 vs. 10 to 13, and E). This result suggested that the Nt-Ser residue of wild-type SPPck $1_{3 \mathrm{f}}$ has to be removed (causing a delay) before the recognition of resulting $\mathbf{P}-\mathrm{Pck} 1_{3 \mathrm{f}}$ by Gid4. Degradation curves in this and other figures that describe Tc/PRT chases were determined for the actual chase data shown. All Tc/PRT chases of this study were carried out at least twice (usually thrice), with results differing by $10 \%$ or less.

The $S$. cerevisiae genome encodes $\sim 20$ aminopeptidases, either putative or confirmed ones (https://www.yeastgenome. org/search?q=aminopeptidases\&is_quick=true). To decrease, if possible, the number of degradation assays and genetic tests with different aminopeptidase mutants, we attempted to "guess," using previously published analyses, either confirmed or putative aminopeptidases as being a priori more or less likely to play a role in the trimming of Xaa-Pro protein substrates. Five aminopeptidases that were chosen, thereby, for testing were Ape2, Ape4, Icp55, Yfr006, and Fra1 (the latter two were putative aminopeptidases; https://www.yeastgenome.org/search? $\mathrm{q}=$ aminopeptidases\&is_quick $=$ true $)$. Initial tests showed that null mutants in the Ape 2 and/or Ape 4 aminopeptidases did not stabilize the normally short-lived AP-Aro $10_{3 \mathrm{f}}$ and $\mathbf{S P}-\mathbf{P c k} 1_{3 \mathrm{f}}$. Since "relevant" aminopeptidases may functionally overlap (in such cases, single-gene mutants may be less informative), we also constructed double-mutant strains such as [fra1 $\Delta$ fro06w $\Delta$ ], which lacked both Fra1 and Yfr006w, two putative (uncharacterized) aminopeptidases. Remarkably, the normally short-lived 


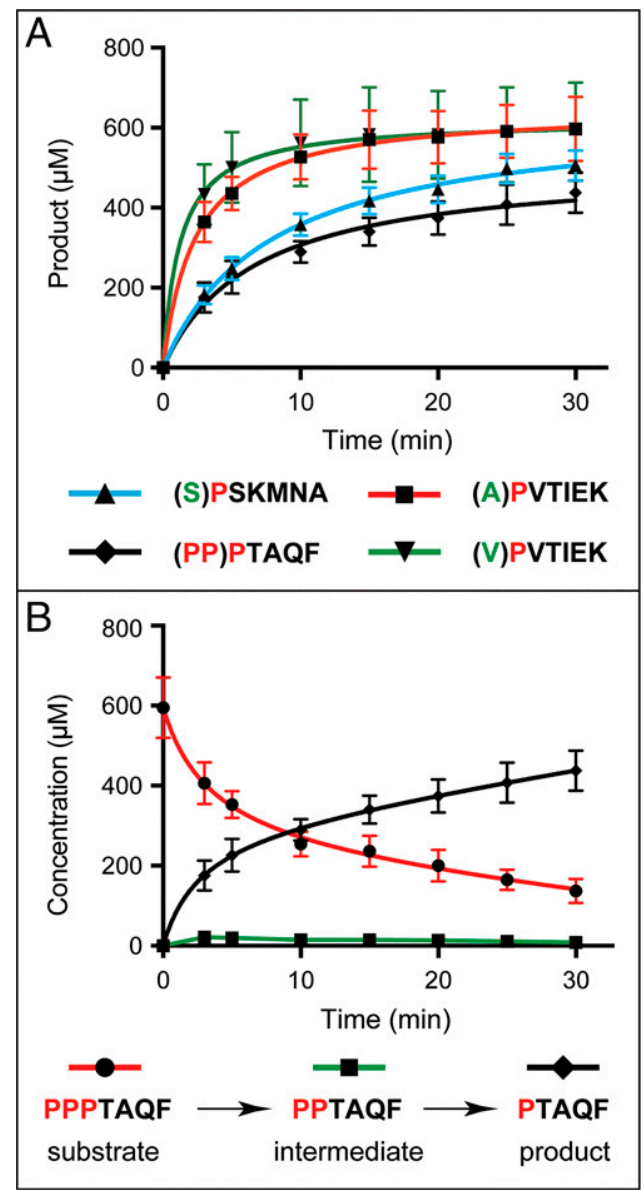

Fig. 2. In vitro enzymatic assays with Fra1 aminopeptidase and peptide substrates. (A) HPLC analyses of proteolysis by Fra1. Peptides (S)PSKMNA, (A)PVTIEK, (PP)PTAQF, and (V)PVTIEK were converted by Fra1 to products through the removal of residues in parentheses. $(B)$ The trimming of PPPTAQF to PTAQF by Fra1 was processive since the levels of the PPTAQF intermediate were negligible or nearly so, indicating that the cleavage that generates PPTAQF was nearly immediately followed by the next and last cleavage.

AP-Aro $10_{3 \mathrm{f}}$ became virtually completely stable in $[$ fra $1 \Delta$ yfroo6w $\Delta]$ cells (Fig. $1 A$, lanes 2 to 5 vs. 6 to 9 , and $B$ ). Degradation of AP-Aro $10_{3 \mathrm{f}}$ requires its Pro at position 2, since AS-Aro10 $3 \mathrm{f}$ was stable in both wild-type and $[$ fral $\Delta$ fro06w $\Delta]$ cells (Fig. $1 A$, lanes 10 to 17 , and $B$ ).

Furthermore and crucially, the normally short-lived AP-Aro $10_{3 \mathrm{f}}$ was found to be virtually completely stable in fra $1 \Delta$ cells, which contained Yfr006w and lacked Fra1 (Fig. $1 C$, lanes 2 to 5 vs. 6 to 9, and D) (SI Appendix, Fig. S2 F, lanes 2 to 5 vs. 6 to 9 , and $G$ ). Thus, Fra1 is essential for the in vivo degradation of AP-Aro10 $3 \mathrm{f}$ and is either the sole or (at least) the major aminopeptidase that trims AP proteins. In addition and tellingly, Fra1 was found to be unnecessary for the degradation of P-Aro $10_{3 \mathrm{f}}$, the engineered Nt-Pro-bearing derivative of APAro $10_{3 \mathrm{f}}$, indicating that Fra1 was required specifically for the conversion of AP-Aro $10_{3 \mathrm{f}}$ to P-Aro10 ${ }_{3 \mathrm{f}}$ (SI Appendix, Fig. S2 F, lanes 10 to 17 , and $G$ ). As one would already expect from the cited results with $\mathbf{S P}-\mathbf{P c k} 1_{3 \mathrm{f}}$ vs. (engineered) $\mathbf{P}-\mathbf{P}$ ck $1_{3 \mathrm{f}}$ (SI Appendix, Fig. S2 $D$ and $E$ ), the engineered Nt-Pro-bearing $\mathbf{P}$ Aro10 $_{3 \mathrm{f}}$ was shorter-lived than AP-Aro10 ${ }_{3 \mathrm{f}}$ (SI Appendix, Fig. $\mathrm{S} 2 F$, lanes 2 to 5 , and $G$ ). Cultures of fral $\Delta$ cells grew at rates similar to those of congenic wild-type cultures.

Aminopeptidase Assays with Purified Fra1. Until the present study, S. cerevisiae Fra1 was a putative aminopeptidase. To

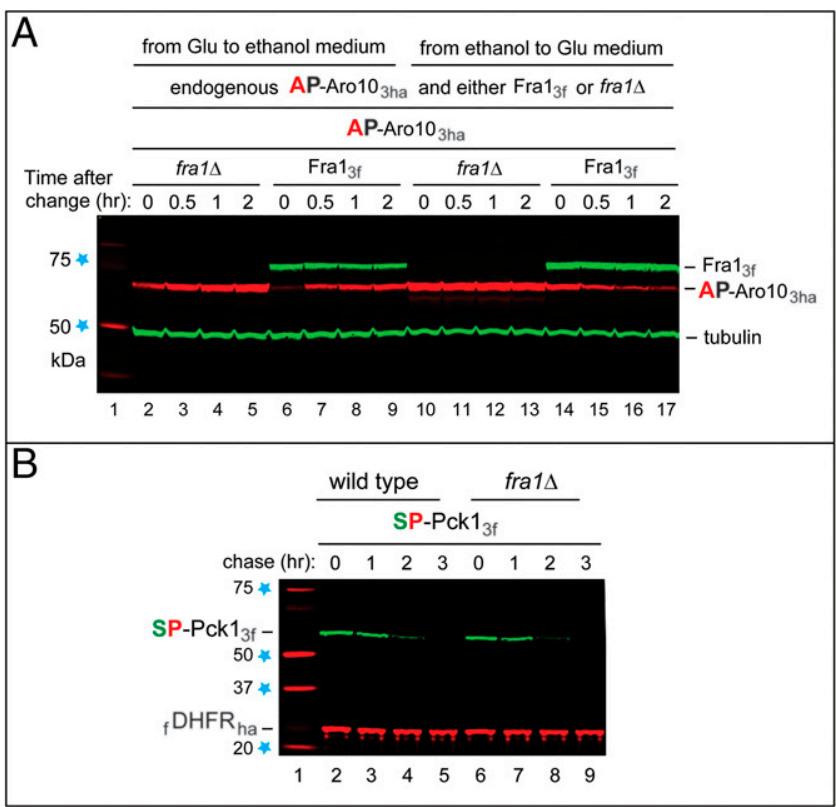

Fig. 3. S. cerevisiae strains in which AP-Aro $10_{3 \text { ha }}$ and $\mathrm{Fra1}_{3 \mathrm{f}}$ are expressed from their endogenous genes and native promoters. (A) SI Appendix, SI Materials and Methods describes the construction of these strains. Immunoblotting (IB) with antibody to tubulin was used to verify loading uniformity. Lane 1, kilodalton markers. Lanes 2 to 5 , the glucose (Glu) $\rightarrow$ ethanol transition, with fra $1 \Delta$ cells expressing AP-Aro $10_{3 \text { ha, }}$ was followed by IB for $2 \mathrm{~h}$ postshift. Lanes 6 to 9 , the same as lanes 2 to 5 but with wildtype cells (AP-Aro10 $1_{3 \text { ha }}$ and Fra $1_{3 f}$ ). Lanes 10 to 13 , the same as lanes 2 to 5 , but cells were subjected to the ethanol $\rightarrow$ glucose (Glu) transition after $20 \mathrm{~h}$ in ethanol-containing medium ( $S I$ Appendix, SI Materials and Methods). Lanes 14 to 17 , the same as lanes 10 to 13 but with cells expressing both $\mathrm{AP}-\mathrm{Aro1}_{3 \mathrm{ha}}$ and $\mathrm{Fra1}_{3 \mathrm{f}}$. The text has additional details. (B) SP-Pck $1_{3 f}$ remains short-lived in the absence of Fra1 aminopeptidase. Lane 1, kilodalton markers. Lanes 2 to 5, Tc/PRT chase with SP-Pck $1_{3 f}$ for 0 to $3 \mathrm{~h}$ at $30^{\circ} \mathrm{C}$ in wild-type cells. Lanes 6 to 9 , the same as lanes 2 to 5 but in fra $1 \Delta$ cells. Note the continued instability of SP-Pck $1_{3 f}$ in the absence of Fra1. Blue stars on the left denote kilodalton markers.

explore its enzymatic activity and specificity, $\mathrm{Ct}-\mathrm{His}_{8}$-tagged Fra1 $1_{\text {His8 }}$ was expressed in Escherichia coli; purified ; and incubated with peptides APVTIEK, VPVTIEK, SPSKMNA, and PPPTAQF. Except for VPVTIEK, their sequences were identical to Nt-sequences of AP-Aro10, SP-Pck1, and PPP-Azf1, respectively $(38,46)$. Incubations were followed by high performance liquid chromatography (HPLC) fractionation and quantification of separated substrates and products (Fig. 2). Purified Fra1 $_{\text {His } 8}$ removed Nt-Ala from APVTIEK and Nt-Val from VPVTIEK (yielding PVTIEK) (Fig. 2A). Fra1 also removed Nt-Ser from SPSKMNA (yielding PSKMNA), with the activity toward Nt-Ser-bearing SPSKMNA being significantly lower than the one toward Nt-Ala-bearing APVTIEK (Fig. 2A). Fra1His8 also cleaved off Pro residues from the PPPTAQF peptide, stopping at PTAQF (Fig. $2 B$ ). The trimming of PPPTAQF to PTAQF by Fra1 was processive since the levels of PPTAQF intermediate were negligible or nearly so, indicating that the cleavage that generates PPTAQF was nearly immediately followed by the next and last cleavage (Fig. $2 B$ ).

Expression of C-Terminally Tagged AP-Aro10 and Fra1 from Their Endogenous Genes and Native Promoters. Activity of the $S$. cerevisiae Pro/N-degron pathway depends on whether cells are grown in the presence of glucose or alternatively, in the presence of a nonfermentable carbon source such as ethanol or acetate (38, 64-66). In particular, the Pro/ $\mathrm{N}$-degron pathway is up-regulated during transitions from, for example, ethanol to 


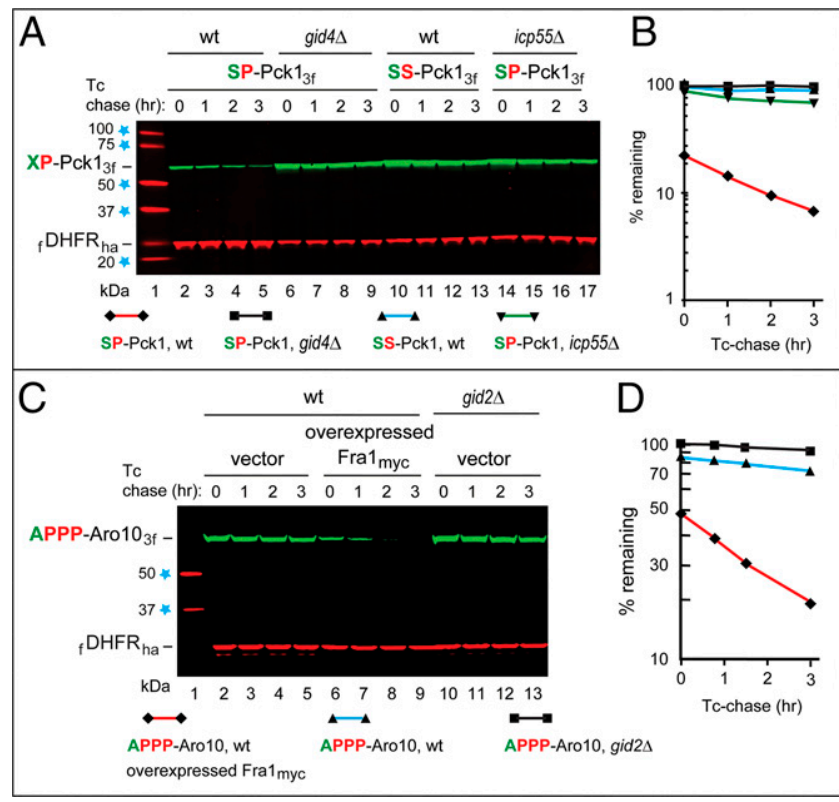

Fig. 4. The bulk of SP-Pck1 degradation requires Icp55 aminopeptidase, and the effect of overexpressed Fra1 aminopeptidase on the degradation of APPP-Aro10. (A) Lane 1, kilodalton markers. Lanes 2 to 5, Tc/PRT chase of SP-Pck $1_{3 f}$ in wild-type (wt) cells. Lanes 6 to 9 , the same as lanes 2 to 5 but in gid $4 \Delta$ cells. Lanes 10 to 13 , the same as lanes 2 to 5 but with SSPck1 $1_{3 f}$. Lanes 14 to 17 , the same as lanes 2 to 5 but in icp55 cells. (B) Quantification of data in $A$. (C) Lane 1, kilodalton markers. Lanes 2 to 5 , Tc/PRT chase of APPP-Aro $10_{3 \mathrm{f}}$ in wt cells. Lanes 6 to 9, the same as lanes 2 to 5 but in wt cells overexpressing Fra $1_{\text {myc }}$ from the P PuP1 promoter on a high-copy plasmid. Lanes 10 to 13 , the same as lanes 2 to 5 but in gid2A cells lacking the active GID Ub ligase. (D) Quantification of data in $C$. Regarding the absence of SD bars in $B$ and $D$ : a detailed explanation is in the text and in Fig. 1. Blue stars on the left denote kilodalton markers.

glucose (Concluding Remarks). To explore impacts of carbon source transitions on AP-Aro10 and Fra1, their endogenous genes were tagged, using standard reverse genetic methods (67), with DNA segments that encoded Ct-triple-ha (3ha) and Ct-triple-flag, respectively. The resulting $S$. cerevisiae strains yCSJ90 (AP-Aro10 3 ha in the fral $\Delta$ background) and yCSJ92 (AP-Aro10 3 ha and Fra1 $_{3 f}$ ) (SI Appendix, Table S1) expressed either AP-Aro $10_{3 \text { ha }}$ or both AP-Aro $10_{3 \text { ha }}$ and Fra $1_{3 \mathrm{f}}$ from their endogenous genes and native promoters. The endogenous Fra $_{3 \mathrm{f}}$ aminopeptidase was long-lived on both ethanol and glucose media (Fig. 3A). The endogenous AP-Aro10 3 ha was up-regulated upon glucose $\rightarrow$ ethanol transitions, more strongly in fral $\Delta$ cells, since AP-Aro10 3 ha was stable in the absence of Fra1 (Figs. $1 C$ and $D$ and $3 A$, lanes 2 to 9 and SI Appendix, Fig. S2 $F$ and $G$ ). The endogenous AP-Aro10 3 ha was degraded upon ethanol $\rightarrow$ glucose transitions [which up-regulate the Pro/N-degron pathway $(38,39,43)]$ but was stable in fral $\Delta$ cells (Fig. $3 \mathrm{~A}$, lanes 10 to 17 ), in agreement with the understanding that emerged from entirely different approaches (Figs. 1, 2, and $3 A$ and SI Appendix, Fig. S2).

Aminopeptidase Activity of Fra1 Is Required for Degradation of AP-Aro10. To determine whether the enzymatic activity of Fra1 (Fig. 2) was actually required for the in vivo degradation of AP-Aro10, we used the above $S$. cerevisiae strains that expressed AP-Aro $10_{3 \text { ha }}$ from its endogenous gene and native promoter and either lacked $($ fra $1 \Delta)$ or contained the endogenous wild-type Fra1 (Fig. 3A). Since AP-Aro $10_{3 \text { ha }}$ was expressed, in these experiments, from the endogenous $A R O 10$ locus, the Tc/PRT method (SI Appendix, Fig. S2C) could not be used, making it necessary to employ cycloheximide chases (SI Appendix, Fig. S3).

\begin{tabular}{|c|c|c|c|c|c|}
\hline$A$ & Pck1 & B & Aro10 & C & Acs1 \\
\hline S. cer: & (M) SPSKMN & S. cer: & (M) APVTIE & S. cer: & (M) SPSAVQ \\
\hline S. par: & (M) SPSKMN & C. gla: & (M) APVTLD & C. gla: & (M) SPSAIA \\
\hline S. mik: & (M) SPSKMN & K. afr: & (M) APVTLH & K. afr: & (M) SPSSTI \\
\hline S. bay: & (M) SPSKMN & S. arb: & (M) APVTID & K. lac: & (M) SPAVDT \\
\hline S. cas: & (M) SPIKVS & T. bla: & (M) $\mathrm{A}$ & S. kud: & (M) SPSAVQ \\
\hline S. klu: & (M) SPSKTY & pol: & $\mathrm{TH}$ & el: & M) SPSAVP \\
\hline K. lac: & (M) SPTKTQ & C. mal: & IQTS & N. cas: & (M) CPTASS \\
\hline A. gos: & (M) SPSKVY & C. ort: & (M) APVRET & T. bla: & (M) PAWKDT \\
\hline A. nid: & (M) APGANI & K. pha: & $\mathrm{KQD}$ * & F. & DNIED \\
\hline C. alb: & (M) APPTAV & K. pha: & PDI * & C. 1 & (M) PESTQH \\
\hline D. han: & (M) TPPTAV & D. han: & (M) $A$ & K. pha: & LDNEH \\
\hline C. lus: & (M) VPPVAV & Z. rou: & (M) $\mathrm{C}$ & C. lus: & (M) PEHLAM \\
\hline Y. lip: & (M) SPPNQT & K. lac: & QLD & L. elo: & EQKNT \\
\hline L. elo: & (M) APPAVE & L. thi: & (M) SPIGIL & M. cir: & (M) PHSTEN \\
\hline C. tro: & (M) APPPTA & S. sti: & (M) TPVQET & R. del: & (M) PMVPEA \\
\hline C. par: & (M) SPPPPA & S. pas: & (M) TPVIET & A. gyp: & SLHPQ \\
\hline C. gla: & (M) PSKSSV & C. dub: & (M) TPIQQT & M. ory & (M) TPEASK \\
\hline fruit fly: & (M) PELIEQ & S. kud: & (M) TPVTIN & fruit fly: & (M) TPEKSV \\
\hline mouse: & (M) PPQLHN & C. alb: & (M) TPIQQT & mouse: & MEVHELFR \\
\hline human: & (M) PPQLQN & M. gui: & (M) VPIATN & human: & MQAHELFR \\
\hline
\end{tabular}

Fig. 5. Evolution of Nt sequences in Pck1 (A), Aro10 (B), and Acs1 (C). [The latter enzyme is Ac-CoA synthetase (46).] (M) denotes the initially present Nt-Met residue, which is cotranslationally removed if a residue at position 2, the one to become $\mathrm{Nt}$, is not larger than Val (73). Note extensive but apparently selection-constrained transitions, on evolutionary timescales, between the Nt-P configuration (which is directly recognized by Gid4-GID [A]) and other Pro-containing Nt-motifs, including SP, AP, TP, CP, PP, TPP, VPP, SPP, APP, APPP, and SPPPP. All of these Nt-sequences are either known or expected to be substrates of Fra1 and/or Icp55 aminopeptidases, which are conserved from fungi to animals and plants. These enzymes would trim the above Nt-motifs, yielding a single Nt-P, the main recognition determinant of a Pro/N-degron (also in the text).

As expected, the endogenous AP-Aro10 3 ha was short-lived in wild-type Fra1-containing cells but long-lived in fral $\Delta$ cells (SI Appendix, Fig. S3, lanes 2 to 9). AP-Aro10 3 ha became destabilized in the latter setting upon overexpression (from the $\mathrm{P}_{\text {CUP1 }}$ promoter on a high-copy plasmid) of the wild-type Ct-myctagged Fra1 $1_{\text {myc }}$ (SI Appendix, Fig. S3, lanes 10 to 13). Even individually (let alone together), the Glu660 $\rightarrow$ Ala $($ E660A) mutation and the His531 $\rightarrow$ Ala $($ H531A) mutation in the metal binding site and the substrate binding segment of Fra1 analogs in other organisms (SI Appendix, Fig. S4), respectively, have been shown to suffice for abrogating virtually $100 \%$ of aminopeptidase activity (ref. 68 and references therein). Tellingly and in agreement with this understanding, AP-Aro10 3 ha continued to be long-lived upon overexpression (confirmed by immunoblotting) in frals cells of three otherwise identical but mutant Fra1 proteins: Fra1 $1_{\text {myc }}^{\mathrm{E} 660 \mathrm{~A}}$, Fra1 $1_{\mathrm{myc}}^{\mathrm{H} 531 \mathrm{~A}}$, or the doublemutant Fra $1_{\text {myc }}^{\mathrm{H} 531 \mathrm{~A}}$, E660A $(S I$ Appendix, Fig. S3, lanes 14 to 25). Thus, the enzymatic activity of Fra1 (i.e., its ability to trim XaaPro Nt-sequences) (Fig. 2) is essential for the in vivo degradation of XP proteins such as AP-Aro $10_{3 \text { ha }}$.

Before the present study, Fra1 was a putative aminopeptidase. Together, these results (Figs. 1, 2, and $3 A$ and $S I$ Appendix, Figs. S2-S4) indicated, first, that $S$. cerevisiae Fra1 is an aminopeptidase with a distinct substrate specificity and long in vivo half-life and second, that the presence of Fra1 and its aminopeptidase activity are essential for the in vivo degradation of AP-Aro10 through the removal of its Nt-Ala residue, a step that yields P-Aro10 and initiates its GID-dependent processive degradation (Fig. 6).

S. cerevisiae Fra1 was originally identified as a contributor, through an unknown mechanism, to regulation of the iron uptake transcriptional system and also as a subunit of specific antioxidant protein complexes $(69,70)$. Connections between 


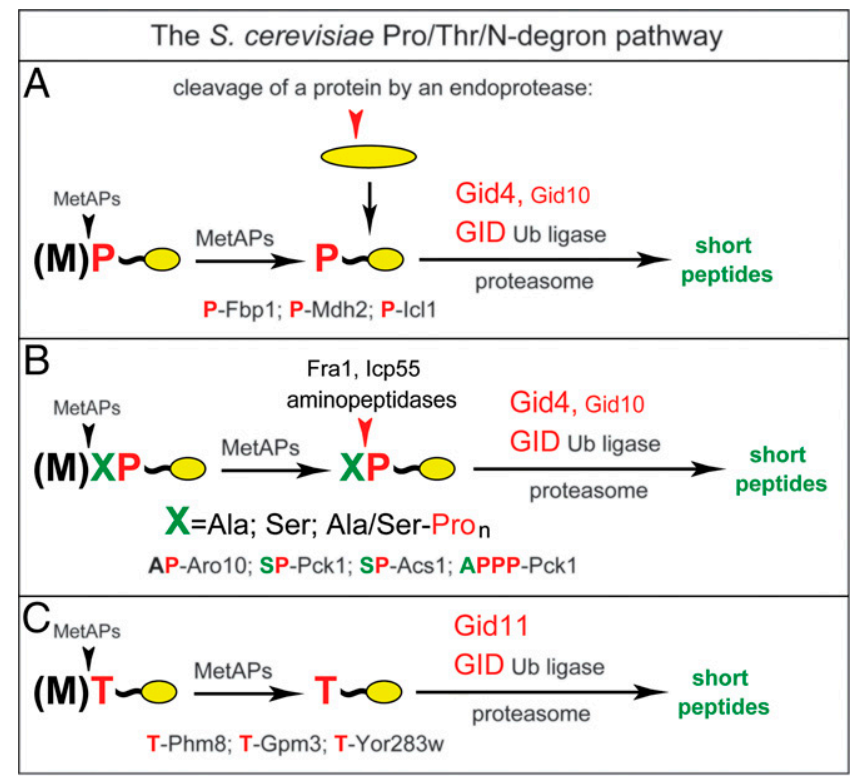

Fig. 6. The $S$. cerevisiae Pro/Thr/N-degron pathway. This diagram summarizes the initially discovered part of the pathway $(A)(38)$, the part that involves aminopeptidases identified in the present study $(B)$, and the 2021 advance by Khmelinskii and colleagues (47) that identified Gid11 (Ylr149c) as an alternative (Gid4-replacing) subunit of the GID ligase that recognizes $\mathrm{Nt}-\mathrm{Thr}(C)$. (A) Nt-Pro-bearing proteins are formed through the cotranslational removal of Nt-Met and are targeted for polyubiquitylation and proteasomal degradation through the recognition of Nt-Pro by Gid4-GID (38, 46). In transitions that are relevant to all three panels, a pathway's substrate can also be formed through a cleavage by an endoprotease, yielding (either directly or after further processing) a Ct-fragment bearing a single Nt-Pro. (B) As discovered in the present study (Figs. 1-5), proteins such as SP-Pck1, AP-Aro10, and APPP-Aro10 can be trimmed by aminopeptidases Fra1 and/or Icp55 to yield substrates bearing a single Nt-Pro residue and targeted for degradation by Gid4-GID. (C) Substrates bearing Nt-Thr, such as T-Phm8, TGpm3, and T-Yor283w, can be targeted by Gid11-GID (47).

these attributes of Fra1 and its function as an aminopeptidase in the Pro/N-degron pathway (Fig. 6) remain to be understood.

The Bulk of SP-Pck1 Degradation Requires Icp55 Aminopeptidase. In contrast to $\mathbf{A P}-A$ ro $10_{3 \mathrm{f}}$, SP-Pck $1_{3 \mathrm{f}}$ remained short-lived in fral $\Delta$ cells (Fig. $3 B$ ). Attempting to identify a particularly relevant Nt-Ser-removing aminopeptidase, we considered Icp55, which is imported into mitochondria but is also present in the cytosol/nucleus. Icp55 can cleave a protein before some nonPro residues at position P1', but its activity is much higher with Pro at position P1' (69-72). Yeast Icp55 can cleave, in the nucleus, the YSP-Nfs1 cysteine desulfurase, producing, sequentially, SP-Nfs1 and P-Nfs1. The Nt-YSP $\rightarrow$ YAP mutation in Nfs1 shifts the Icp55 cleavage site from $\mathbf{Y S} \downarrow \mathbf{P}$ to $\mathbf{Y} \downarrow \mathbf{A P}$, indicating a preference of Icp55 for Ser at position P1 (71).

Remarkably, the normally short-lived SP-Pck $1_{3 \mathrm{f}}$ became long lived in ipc55 $\Delta$ cells (Fig. $4 A$ and $B$ ). While AP-Aro10 ${ }_{3 \mathrm{f}}$ was virtually completely stabilized in fra $1 \Delta$ cells (Fig. $1 C$ and $D$ and SI Appendix, Fig. S2 $F$ and $G$ ), stabilization of SP-Pck $1_{3 \mathrm{f}}$ in icp55 $\Delta$ cells was very strong but not as complete, suggesting that either Fra1 (which can remove Nt-Ser, albeit significantly more slowly than Nt-Ala) (Fig. $2 A$ ) or another aminopeptidase can marginally contribute to removal of Nt-Ser, in addition to the major input by Icp55 (Fig. $4 A$ and $B$ ).

Evolution of Nt-Sequences in Xaa-Pro Proteins. The evolution of Nt-sequences in Pck1, Aro10, and Acs1 $(38,46)$ is illustrated in Fig. 5. [The $S$. cerevisiae acetyl-CoA synthetase SP-Acs1 has been identified as a substrate of the Pro/N-degron pathway (46).]
Among fungi/yeasts, Pck1 is SP-Pck1 in S. cerevisiae, AP-Pck1 in Aspegillus nidulans, APP-Pck1 in Lodderomyces elongisporus, APPP-Pck1 in Candida tropicalis, and SPPPP-Pck1 in Candida parapsilosis, while in the fly Drosophila melanogaster and in humans/mice, Pck1 is P-Pck1 and PP-Pck1, respectively (Fig. 5).

Can Nt-sequence of, for example, $C$. tropicalis APPP-Pck1 generate an active Pro/N-degron in vivo? We replaced Nt-AP of AP-Aro $10_{3 \mathrm{f}}$ with Nt-APPP and carried out Tc/PRT chases in the absence or presence of overexpressed Fra1 $1_{\text {myc }}$. The degradation of APPP-Aro $10_{3 \mathrm{f}}$ in wild-type cells that did not overexpress Fra1myc was detectable but quite slow (Fig. $4 C$ and $D$ ). In contrast, APPP-Aro $10_{3 \mathrm{f}}$ was much shorter-lived in cells that overexpressed Fra1, with major effects on the slope of the APPP-Aro10 $3 \mathrm{f}$ degradation curve and its time-zero level (Fig. $4 C$ and $D$ ).

Thus, reiterated Nt-proximal Pro residues of at least some proteins that are cited in Fig. 5 can function as components of Pro/N-degrons. Such Pro residues were retained and often amplified during evolution (Fig. 5), possibly to modulate degradation rates of proteins bearing these Nt-sequences. Natural selection apparently constrained genetic drifts either to Procontaining Nt-motifs that can be trimmed by Fra1-type aminopeptidases (generating a single Nt-Pro) or to Nt-sequences that bear a single Nt-Pro from the start and therefore, can be directly recognized by Gid4-GID. Examples of the latter kind are the Pck1 proteins of Candida glabrata and the fruit fly and some of the cited fungal Acs1 proteins (Fig. 5).

Concluding Remarks. GID-mediated processes include a previously studied but incompletely understood dichotomy between the GID/proteasome-mediated degradation of gluconeogenic enzymes (Fig. 6) and "alternative" degradation of these enzymes through an autophagy-related pathway termed VID (vacuole import and degradation) (refs. 64-66 and references therein). If $S$. cerevisiae is grown either in the presence of glucose or on a nonfermentable carbon source such as ethanol or acetate (in the latter cases, for less than $\sim 12 \mathrm{~h}$ before returning cells to glucose), the relative activity of VID is negligible. However, a much longer growth on, for example, ethanol or acetate results (after return to glucose) in the VID-mediated degradation of gluconeogenic enzymes (64-66). Remarkably, both the proteasome-mediated and VID-mediated degradation of these enzymes require Gid4 Pro/N-recognin and other subunits of the GID Ub ligase. Detailed reasons for the involvement of two distinct mechanisms for the conditional degradation of proteins that bear Pro/N-degrons are unknown. How the ability of Gid4 to recognize Pro/N-degrons is employed by the VID pathway (as distinguished from the proteasome) and whether the two aminopeptidases of the present study (Fig. 6B) play a role in VID remain to be determined as well.

Evolution repeatedly exploited specific topological and chemical features of N-termini, creating numerous Ntmodifications. Many of them and the initial (unmodified) Ntresidues as well evolved to function as specific $\mathrm{N}$-degrons (Fig. 6 and SI Appendix, Fig. S1). The GID Ub ligase and the Fra1 and Icp55 aminopeptidases are conserved in evolution (SI Appendix, Fig. S4) (refs. 38-46 and 68-72 and references therein), making the insights of this study relevant to eukaryotes from fungi to animals and plants. In addition to discovering the mechanism of targeting of Xaa-Pro proteins, these results have also expanded the diversity of substrates of the Pro/N-degron pathway.

\section{Materials and Methods}

Further information is in SI Appendix, SI Materials and Methods.

Antibodies, Other Reagents, Yeast Strains, and Genetic Techniques. S. cerevisiae strains used in this work are described in SI Appendix, Table S1. Standard techniques were used for strain construction and transformation. 
Construction of Plasmids. The plasmids are described in SI Appendix, Table S2. Construction details are described in SI Appendix, SI Materials and Methods.

Tc-Based PRT Chase Assays and Immunoblotting. The PRT is described in $\mathrm{SI}$ Appendix, Fig. 2C. Details of TC/PRT chase experiments are described in $\mathrm{SI}$ Appendix, SI Materials and Methods.

Edman-Based Nt-Sequencing of Isolated AP-Aro $10_{3 f}$ and SP-Pck1 $1_{3 f}$. Details of Edman-based Nt sequencing of AP-Aro $1_{3 f}$ and SP-Pck $1_{3 f}$ are described in $\mathrm{SI}$ Appendix, SI Materials and Methods.

Expression and Purification of Yeast Fra1 $1_{\text {Hiss }}$ Aminopeptidase. S. cerevisiae Fra1 $1_{\text {Hiss }}$ was expressed in E. coli and purified as described in SI Appendix, SI Materials and Methods.

1. A. Hershko, A. Ciechanover, A. Varshavsky, The ubiquitin system. Nat. Med. 6, 1073-1081 (2000)

2. A. Varshavsky, Discovery of cellular regulation by protein degradation. J. Biol. Chem. 283, 34469-34489 (2008).

3. D. Finley, H. D. Ulrich, T. Sommer, P. Kaiser, The ubiquitin-proteasome system of Saccharomyces cerevisiae. Genetics 192, 319-360 (2012).

4. V. Vittal, M. D. Stewart, P. S. Brzovic, R. E. Klevit, Regulating the regulators: Recent revelations in the control of E3 ubiquitin ligases. J. Biol. Chem. 290, 21244-21251 (2015)

5. C. H. Ji, Y. T. Kwon, Crosstalk and interplay between the ubiquitin-proteasome system and autophagy. Mol. Cells 40, 441-449 (2017).

6. C. Pohl, I. Dikic, Cellular quality control by the ubiquitin-proteasome system and autophagy. Science 366, 818-822 (2019).

7. D. Balchin, M. Hayer-Hartl, F. U. Hartl, In vivo aspects of protein folding and quality control. Science 353, aac4354 (2016)

8. Z. Sun, J. L. Brodsky, Protein quality control in the secretory pathway. J. Cell Biol. 218, 3171-3187 (2019)

9. N. Zheng, N. Shabek, Ubiquitin ligases: Structure, function, and regulation. Annu. Rev. Biochem. 86, 129-157 (2017)

10. R. J. Deshaies, C. A. P. Joazeiro, RING domain E3 ubiquitin ligases. Annu. Rev. Biochem. 78, 399-434 (2009).

11. M. Rape, Ubiquitylation at the crossroads of development and disease. Nat. Rev. Mol. Cell Biol. 19, 59-70 (2018)

12. T. E. T. Mevissen, D. Komander, Mechanisms of deubiquitinase specificity and regulation. Annu. Rev. Biochem. 86, 159-192 (2017)

13. E. R. Watson, N. G. Brown, J. M. Peters, H. Stark, B. A. Schulman, Posing the APC/C E3 ubiquitin ligase to orchestrate cell division. Trends Cell Biol. 29, 117-134 (2019).

14. J. A. M. Bard et al., Structure and function of the $26 \mathrm{~S}$ proteasome. Annu. Rev. Biochem. 87, 697-724 (2018).

15. E. Sakata, M. R. Eisele, W. Baumeister, Molecular and cellular dynamics of the $26 \mathrm{~S}$ proteasome. Biochim. Biophys. Acta. Proteins Proteomics 1869, 140583 (2021).

16. D. Finley, M. A. Prado, The proteasome and its network: Engineering for adaptability. Cold Spring Harb. Perspect. Biol. 12, a033985 (2020)

17. G. A. Collins, A. L. Goldberg, The logic of the $26 \mathrm{~S}$ proteasome. Cell 169, 792-806 (2017).

18. H. Yu, A. Matouschek, Recognition of client proteins by the proteasome. Annu. Rev. Biophys. 46, 149-173 (2017).

19. L. Budenholzer, C. L. Cheng, Y. Li, M. Hochstrasser, Proteasome structure and assembly. J. Mol. Biol. 429, 3500-3524 (2017)

20. A. Varshavsky, N-degron and C-degron pathways of protein degradation. Proc. Natl. Acad. Sci. U.S.A. 116, 358-366 (2019)

21. A. Bachmair, D. Finley, A. Varshavsky, In vivo half-life of a protein is a function of its amino-terminal residue. Science 234, 179-186 (1986).

22. A. Bachmair, A. Varshavsky, The degradation signal in a short-lived protein. Cell $\mathbf{5 6}$, 1019-1032 (1989).

23. G. C. Turner, F. Du, A. Varshavsky, Peptides accelerate their uptake by activating a ubiquitin-dependent proteolytic pathway. Nature 405, 579-583 (2000).

24. H. Rao, F. Uhlmann, K. Nasmyth, A. Varshavsky, Degradation of a cohesin subunit by the $\mathrm{N}$-end rule pathway is essential for chromosome stability. Nature 410, 955-959 (2001)

25. Y. T. Kwon et al., An essential role of $\mathrm{N}$-terminal arginylation in cardiovascular development. Science 297, 96-99 (2002).

26. A. Varshavsky, The $\mathrm{N}$-end rule pathway and regulation by proteolysis. Protein Sci. 20, 1298-1345 (2011)

27. T. Tasaki, S. M. Sriram, K. S. Park, Y. T. Kwon, The N-end rule pathway. Annu. Rev. Biochem. 81, 261-289 (2012).

28. J. G. Kim et al., Signaling pathways regulated by UBR box-containing E3 ligases. Int. J. Mol. Sci. 22, 8323 (2021).

29. N. Winter, M. Novatchkova, A. Bachmair, Cellular control of protein turnover via the modification of the amino-terminus. Int. J. Mol. Sci. 22, 3545 (2021).

30. R. T. Timms, I. Koren, Tying up loose ends: The N-degron and C-degron pathways of protein degradation. Biochem. Soc. Trans. 48, 1557-1567 (2020).
In Vitro Assay for Fra1 Aminopeptidase Activity. Aminopeptidase activity of purified Fra $1_{\text {Hiss }}$ was assayed using synthetic peptides and HPLC fractionation (as well as quantification) of substrates and products. Details are described in SI Appendix, SI Materials and Methods.

Data Availability. All relevant data in the paper are entirely available through both text and figures in the manuscript and SI Appendix.

ACKNOWLEDGMENTS. We thank members of the laboratories of H.K.S. and A.V., particularly Jin Seok Shin for initial preparation and analysis of Pck1, and also Chang-Seok Lee and Cheol-Sang Hwang (Postech, Pohang, Korea) for some of yeast strains used in this study. This work was supported by National Research Foundation of Korea Grants NRF-2020R1A2C3008285 (to H.K.S.) and NRF-2020R1A5A1019023 (to H.K.S.) and by NIH Grants DK039520 (to A.V.) and GM031530 (to A.V.).

31. I. Koren, et al., The eukaryotic proteome is shaped by E3 ubiquitin ligases targeting C-terminal degrons. Cell 173, 1622-1635.e1614 (2018).

32. M. J. Holdsworth, J. Vicente, G. Sharma, M. Abbas, A. Zubrycka, The plant $\mathrm{N}$-degron pathways of ubiquitin-mediated proteolysis. J. Integr. Plant Biol. 62, 70-89 (2020)

33. C. S. Hwang, A. Shemorry, A. Varshavsky, N-terminal acetylation of cellular proteins creates specific degradation signals. Science 327, 973-977 (2010).

34. A. Shemorry, C. S. Hwang, A. Varshavsky, Control of protein quality and stoichiometries by $\mathrm{N}$-terminal acetylation and the $\mathrm{N}$-end rule pathway. Mol. Cell 50, 540-551 (2013).

35. H. Aksnes, R. Ree, T. Arnesen, Cotranslational, posttranslational, and noncatalytic roles of N-terminal acetyltransferases. Mol. Cell 73, 1097-1114 (2019).

36. K. I. Piatkov, C. S. Brower, A. Varshavsky, The N-end rule pathway counteracts cel death by destroying proapoptotic protein fragments. Proc. Natl. Acad. Sci. U.S.A. 109, E1839-E1847 (2012)

37. C. S. Brower, K. I. Piatkov, A. Varshavsky, Neurodegeneration-associated protein fragments as short-lived substrates of the N-end rule pathway. Mol. Cel/ 50, 161-171 (2013).

38. S. J. Chen, X. Wu, B. Wadas, J.-H. Oh, A. Varshavsky, An N-end rule pathway that rec ognizes proline and destroys gluconeogenic enzymes. Science 355, 366 (2017).

39. M. Hämmerle et al., Proteins of isolated mutants and $\mathrm{N}$-terminal proline are essentia for degradation of yeast fructose-1,6-bisphosphatase. J. Biol. Chem. 273, 25000-25005 (1998).

40. C. Dong et al., Molecular basis of GID4-mediated recognition of degrons for the Pro/ $\mathrm{N}$-end rule pathway. Nat. Chem. Biol. 14, 466-473 (2018).

41. D. A. Dougan, A. Varshavsky, Understanding the Pro/N-end rule pathway. Nat. Chem Biol. 14, 415-416 (2018)

42. A. Melnykov, S. J. Chen, A. Varshavsky, Gid10 as an alternative N-recognin of the Pro/ N-degron pathway. Proc. Natl. Acad. Sci. U.S.A. 116, 15914-15923 (2019).

43. S. J. Chen, A. Melnykov, A. Varshavsky, Evolution of substrates and components of the Pro/N-degron pathway. Biochemistry 59, 582-593 (2020)

44. S. Qiao et al., Interconversion between anticipatory and active GID E3 ubiquitin ligase conformations via substrate receptor assembly. Mol. Cell 77, 150-163.e159 (2020)

45. D. Sherpa et al., GID E3 ligase supramolecular chelate assembly configures multipronged ubiquitin targeting of an oligomeric metabolic enzyme. Mol. Cell 81, 2445-2459.e13 (2021)

46. O. Karayel, A. C. Michaelis, M. Mann, B. A. Schulman, C. R. Langlois, DIA-based systems biology approach unveils E3 ubiquitin ligase-dependent responses to a metabolic shift. Proc. Natl. Acad. Sci. U.S.A. 117, 32806-32815 (2020)

47. K. E. Kong et al., Timer-based proteomic profiling of the ubiquitin-proteasome system reveals a substrate receptor of the GID ubiquitin ligase. Mol. Cell $\mathbf{8 1}$ 2460-2476.e11 (2021).

48. J. M. Kim et al., Formyl-methionine as an $\mathrm{N}$-degron of a eukaryotic $\mathrm{N}$-end rule path way. Science 362, eaat0174 (2018).

49. J. H. Oh, J. Y. Hyun, A. Varshavsky, Control of Hsp90 chaperone and its clients by $\mathrm{N}$-terminal acetylation and the $\mathrm{N}$-end rule pathway. Proc. Natl. Acad. Sci. U.S.A. 114 E4370-E4379 (2017)

50. J. H. Oh, J. Y. Hyun, S. J. Chen, A. Varshavsky, Five enzymes of the Arg/N-degron pathway form a targeting complex: The concept of superchanneling. Proc. Natl. Acad. Sci. U.S.A. 117, 10778-10788 (2020).

51. T. T. M. Vu, A. Varshavsky, The ATF3 transcription factor is a short-lived substrate of the Arg/N-degron pathway. Biochemistry 59, 2796-2812 (2020).

52. T. T. M. Vu, D. C. Mitchell, S. P. Gygi, A. Varshavsky, The Arg/N-degron pathway tar gets transcription factors and regulates specific genes. Proc. Natl. Acad. Sci. U.S.A 117, 31094-31104 (2020)

53. T. Suzuki, A. Varshavsky, Degradation signals in the lysine-asparagine sequence space. EMBO J. 18, 6017-6026 (1999).

54. M. K. Kim, S. J. Oh, B. G. Lee, H. K. Song, Structural basis for dual specificity of yeast $\mathrm{N}$-terminal amidase in the $\mathrm{N}$-end rule pathway. Proc. Natl. Acad. Sci. U.S.A. 113 12438-12443 (2016).

55. W. S. Choi et al., Structural basis for the recognition of $\mathrm{N}$-end rule substrates by the UBR box of ubiquitin ligases. Nat. Struct. Mol. Biol. 17, 1175-1181 (2010). 
56. T. Inobe, S. Fishbain, S. Prakash, A. Matouschek, Defining the geometry of the twocomponent proteasome degron. Nat. Chem. Biol. 7, 161-167 (2011).

57. X. Gao, J. Yeom, E. A. Groisman, The expanded specificity and physiological role of a widespread N-degron recognin. Proc. Natl. Acad. Sci. U.S.A. 116, 18629-18637 (2019).

58. J. Yeom, X. Gao, E. A. Groisman, Reduction in adaptor amounts establishes degradation hierarchy among protease substrates. Proc. Natl. Acad. Sci. U.S.A. 115, E4483-E4492 (2018)

59. K. H. Wang, G. Roman-Hernandez, R. A. Grant, R. T. Sauer, T. A. Baker, The molecular basis of N-end rule recognition. Mol. Cel/ 32, 406-414 (2008).

60. L. Kim et al., Structural basis for the $\mathrm{N}$-degron specificity of $\mathrm{ClpS1}$ from Arabidopsis thaliana. Protein Sci. 30, 700-708 (2021).

61. H. Liu, T. Pfirrmann, The Gid-complex: An emerging player in the ubiquitin ligase league. Biol. Chem. 400, 1429-1441 (2019).

62. J. B. Smith, Peptide sequencing by Edman degradation. eLS 1, 1-3 (2001).

63. J. H. Oh, S. J. Chen, A. Varshavsky, A reference-based protein degradation assay without global translation inhibitors. J. Biol. Chem. 292, 21457-21465 (2017).

64. J. Regelmann et al., Catabolite degradation of fructose-1,6-bisphosphatase in the yeast Saccharomyces cerevisiae: A genome-wide screen identifies eight novel GID genes and indicates the existence of two degradation pathways. Mol. Biol. Cell 14, 1652-1663 (2003).

65. A. A. Alibhoy, H. L. Chiang, Vacuole import and degradation pathway: Insights into a specialized autophagy pathway. World J. Biol. Chem. 2, 239-245 (2011).
66. C. R. Brown, A. B. Wolfe, D. Cui, H. L. Chiang, The vacuolar import and degradation pathway merges with the endocytic pathway to deliver fructose-1,6-bisphosphatase to the vacuole for degradation. J. Biol. Chem. 283, 26116-26127 (2008).

67. B. Andrews, C. Boone, T. N. Davis, S. Fields, Budding Yeast (A Laboratory Manual) (Cold Spring Harbor Press, Cold Spring Harbor, NY, 2016).

68. C. T. Peng et al., Structure-function relationship of aminopeptidase P from Pseudomonas aeruginosa. Front. Microbiol. 8, 2385 (2017).

69. L. Li et al., Dissection of a mitochondria-vacuole signaling pathway reveals a link between oxidative stress and vacuolar iron transport. J. Biol. Chem. 285, 10232-10242 (2010).

70. A. Kumánovics et al., Identification of FRA1 and FRA2 as genes involved in regulating the yeast iron regulon in response to decreased mitochondrial iron-sulfur cluster synthesis. J. Biol. Chem. 283, 10276-10286 (2008)

71. A. Naamati, N. Regev-Rudzki, S. Galperin, R. Lill, O. Pines, Dual targeting of Nfs1 and discovery of its novel processing enzyme, Icp55. J. Biol. Chem. 284, 30200-30208 (2009).

72. F. N. Vögtle et al., Global analysis of the mitochondrial N-proteome identifies a processing peptidase critical for protein stability. Cell 139, 428-439 (2009).

73. Q. Xiao, F. Zhang, B. A. Nacev, J. O. Liu, D. Pei, Protein N-terminal processing: Substrate specificity of Escherichia coli and human methionine aminopeptidases. Biochemistry 49, 5588-5599 (2010). 